\title{
Deprotonation of Isoxazole: A Photoelectron
}

\section{Imaging Study}

\author{
Adam A. Wallace, Yerbolat Dauletyarov, Andrei Sanov* \\ Department of Chemistry and Biochemistry, The University of Arizona, Tucson, Arizona 85721, \\ United States
}

\section{Table of Contents}

Table S1: Geometric parameters of the equilibrium anion and neutral structures resulting from the deprotonation or $\mathrm{H}$-dissociation of isoxazole at the $\mathrm{C} 5, \mathrm{C} 3$, or $\mathrm{C} 4$ positions

Page S2 
Table S1. Geometric parameters of the equilibrium anion and neutral structures resulting from the deprotonation or $\mathrm{H}$-dissociation of isoxazole at the $\mathrm{C} 5, \mathrm{C} 3$, or $\mathrm{C} 4$ positions. The anion structures are shown at the bottom of Figure 4 in the main article. Geometry optimizations were carried out at the CCSD level of theory with the aug-cc-pVTZ basis set. The bond lengths are given in Angstroms and bond angles in degrees.

\begin{tabular}{|c|c|c|c|c|c|c|}
\hline & $\begin{array}{c}\text { C5- } \\
\text { isoxazolide }\end{array}$ & $\begin{array}{c}\text { C5- } \\
\text { isoxazolyl }\end{array}$ & $\begin{array}{c}\text { C3-enolate } \\
\text { anion }\end{array}$ & $\begin{array}{c}\text { C3-enolate } \\
\text { radical }\end{array}$ & $\begin{array}{c}\text { C4- } \\
\text { isoxazolide }\end{array}$ & $\begin{array}{c}\text { C4- } \\
\text { isoxazolyl }\end{array}$ \\
\hline $\mathrm{O}-\mathrm{N}$ & 1.423 & 1.425 & $\mathrm{n} / \mathrm{a}$ & $\mathrm{n} / \mathrm{a}$ & 1.387 & 1.394 \\
\hline $\mathrm{N}-\mathrm{C} 3$ & 1.307 & 1.306 & 1.166 & 1.161 & 1.315 & 1.307 \\
\hline $\mathrm{C} 3-\mathrm{C} 4$ & 1.418 & 1.429 & 1.414 & 1.405 & 1.453 & 1.417 \\
\hline $\mathrm{C} 4-\mathrm{C} 5$ & 1.395 & 1.350 & 1.399 & 1.451 & 1.365 & 1.345 \\
\hline $\mathrm{C} 5-\mathrm{O}$ & 1.372 & 1.304 & 1.245 & 1.217 & 1.388 & 1.347 \\
\hline $\mathrm{C} 3-\mathrm{H}$ & 1.082 & 1.077 & $\mathrm{n} / \mathrm{a}$ & $\mathrm{n} / \mathrm{a}$ & 1.085 & 1.076 \\
\hline $\mathrm{C} 4-\mathrm{H}$ & 1.079 & 1.072 & 1.080 & 1.081 & $\mathrm{n} / \mathrm{a}$ & $\mathrm{n} / \mathrm{a}$ \\
\hline $\mathrm{C} 5-\mathrm{H}$ & $\mathrm{n} / \mathrm{a}$ & $\mathrm{n} / \mathrm{a}$ & 1.113 & 1.098 & 1.082 & 1.075 \\
\hline$\angle \mathrm{O}-\mathrm{N}-\mathrm{C} 3$ & 103.3 & 104.8 & $\mathrm{n} / \mathrm{a}$ & $\mathrm{n} / \mathrm{a}$ & 104.5 & 106.5 \\
\hline$\angle \mathrm{N}-\mathrm{C} 3-\mathrm{C} 4$ & 112.6 & 113.3 & 177.6 & 178.5 & 117.2 & 110.0 \\
\hline$\angle \mathrm{C} 3-\mathrm{C} 4-\mathrm{C} 5$ & 107.0 & 100.3 & 123.8 & 121.1 & 96.5 & 105.8 \\
\hline$\angle \mathrm{C} 4-\mathrm{C} 5-\mathrm{O}$ & 104.0 & 114.8 & 129.9 & 123.0 & 115.7 & 108.7 \\
\hline$\angle \mathrm{N}-\mathrm{C} 3-\mathrm{H}$ & 118.1 & 118.1 & $\mathrm{n} / \mathrm{a}$ & $\mathrm{n} / \mathrm{a}$ & 115.6 & 119.6 \\
\hline$\angle \mathrm{C} 3-\mathrm{C} 4-\mathrm{H}$ & 126.6 & 130.1 & 117.3 & 118.4 & $\mathrm{n} / \mathrm{a}$ & $\mathrm{n} / \mathrm{a}$ \\
\hline$\angle \mathrm{C} 4-\mathrm{C} 5-\mathrm{H}$ & $\mathrm{n} / \mathrm{a}$ & $\mathrm{n} / \mathrm{a}$ & 112.1 & 115.7 & 133.0 & 134.7 \\
\hline
\end{tabular}

